\section{Response availability and second-order conditioned suppression}

\author{
RONALD P. BYRUM* and DONALD E. JACKSON \\ Eastern Michigan University, Ypsilanti, Mich. 48197
}

Following $8 \mathrm{CER}$ training days, 18 rats were given second-order conditioning trials under one of two conditions. Control Ss had the response lever present during all 10 SOC days. Experimental Ss had the lever absent during the first 3 SOC days $\left(\mathrm{CS}_{1}\right.$ was noise, $\mathrm{CS}_{2}$ was a flashing light). A second experiment with 16 rats reversed the first- and second-order stimuli and gave 5 days of SOC with the lever absent for half the Ss. In both studies, when the lever was returned for all Ss, subsequent SOC days showed no significant differences between groups with respect to either acquisition of SOC or extinction of FOC. These results offer no support to Kamil's (1969) hypothesis that his failure to find a correspondence between first- and second-order conditioned suppression might be due to counterconditioning of $\mathrm{CS}_{2}$ to the food reinforcement obtained during SOC.

The purpose of the present studies was to investigate the role of operant responding as a parameter in second-order conditioning (SOC) of a conditioned emotional response (CER). Recently, Davenport (1966) and Kamil (1968) have demonstrated that SOC can be obtained readily by using conventional conditioned suppression techniques modified for higher order conditioning. In these experiments, the following multistage paradigm was employed: (1) establishment of stabilized operant responding; (2) preexposure to the intended first- and second-order CSs (CS, and $\mathrm{CS}_{2}$, respectively); (3) first-order conditioning (FOC), where $\mathrm{CS}_{1}$ is paired with the US, shock; (4) exposure to $\mathrm{CS}_{2}$ again; and (5) $\mathrm{SOC}$, where $\mathrm{CS}_{2}$ is paired with $\mathrm{CS}_{1}$ in the absence of the original US and SOC measured. As is frequently done, operant responding is concurrent with each stage, providing a baseline for monitoring. Kamil (1969), however, has raised the question of the extent to which second-order conditioned suppression obtained by this paradigm is a function of factors other than the assumed internal classically conditioned emotional response to $\mathrm{CS}_{2}$. He suggested that some aspect of operant responding may be a critical parameter during SOC.

In an attempt to investigate second-order conditioned suppression parametrically, Kamil (1969) found that parameters critical in FOC did not produce corresponding effects during SOC. Two experiments investigated the effects of $\mathrm{CS}_{1}$ and $\mathrm{CS}_{2}$ durations, partial vs continuous shock reinforcement during $\mathrm{FOC}$, and delay vs trace FOC. In all cases, conditioned suppression was clearly obtained. But

* Now at the University of Alabama, University, Ala. 35486 . more importantly, $\mathrm{CS}$, and $\mathrm{CS}_{2}$ suppression were not well correlated during the SOC test phase. $\mathrm{He}$ concluded tentatively that response-produced reinforcement might evoke a competing response via a counterconditioning mechanism which interacts with or completely overshadows the effects produced by the FOC parameters mentioned above. In an attempt to evaluate Kamil's hypothesis, the present research investigated the effect of response present or absent) during early SOC

\section{EXPERIMENT 1}

Although the role of response availability in a CER situation has not been studied extensively, there is evidence that it is an important determinant of both the acquisition and extinction of conditioned suppression, both of which are involved in SOC. Wagner, Siegel, \& Fein (1967), for example, could demonstrate partial reinforcement effects only if barpressing was possible during CER acquisition, suggesting that the operant component was the one mainly affected. These results could be interpreted in terms of accidental response-shock contingencies present during CER training. Consequently, response availability during CS-US pairings may be a potentially important training parameter. Operant responding, therefore, during $\mathrm{CS}_{2} \cdot \mathrm{CS}_{1}$ pairings may have altered acquisition of second-order suppression in Kamil's (1969) studies.

In the only studies to date which have manipulated response availability during CER extinction, Jackson (1970) and Byrum \& Jackson (1970) found that when barpressing was possible during early CER extinetion, it served to facilitate subsequent extinction. The latter study further availability (response lever was either upon subsequent SOC. indicated that reinforcement (water) was the critical determinant of the accelerated extinction. The present studies employed a multistage procedure similar to that used by Davenport (1966) and Kamil (1968), modified to allow evaluation of response availability effects during early SOC

Subjects and Apparatus

The Ss were 18 experimentally naive male Sprague-Dawley rats weighing an average of $365 \mathrm{~g}(\mathrm{~s}=19.1)$ at the beginning of experimentation. The experimental chambers used for barpressing, FOC, and SOC were two identical Foringer Skinner boxes with automated programming. They were housed in sound-attenuating blower-ventilated enclosures isolated from both the home cage area and programming apparatus. Each chamber contained a removable response lever and normally recessed dipper which delivered $0.1 \mathrm{cc}$ of water upon activation. Ambient illumination was provided by a $7-\mathrm{W}, 110-$ to $120-\mathrm{V}$ houselight mounted behind the manipulandum panel. Light entered the chamber through the clear plastic top and elevated grid floor. A continuous 87-dB masking noise was provided by the blower. The $\mathrm{CS}_{1}$ was a 1-min 90-dB clicker; $\mathrm{CS}_{2}$ was a $1-\mathrm{min} 25-\mathrm{W} 110-$ to $120^{-} \mathrm{V}$ light flashing at $1.5 \mathrm{fps}$. The light was mounted behind the manipulandum panel, and its presentation resulted in intermittent increases in ambient illumination within the Skinner box. The US used during FOC was a $0.5-\mathrm{sec}$ 1.0-mA scrambled shock delivered through grid floors.

Procedure

At the start of the experiment, Ss were individually housed with food (Purina Lab Chow) available ad lib throughout the experiment. Each $S$ was randomly assigned a chamber and time of day for training and treatment. The Ss were run daily in $28 / 30$-min sessions, with water available for $5 \mathrm{~min}$ beginning $10 \mathrm{~min}$ after the conclusion of each session.

Preliminary operant training. Following automatic magazine training with 33 "free" reinforcements on a VI 20 -sec schedule, Ss self-trained to a criterion of $50 \mathrm{crf}$. During the next six sessions, barpressing stabilized on a VI 1-min reinforcement schedule.

FOC and $\mathrm{CS}_{2}$ preexposure. With the b a $r$ present and water response-contingent, two clicker $\left(\mathrm{CS}_{1}\right)$-shock pairings were given daily for 8 days. During the first 3 FOC days, $\mathrm{CS}_{1}$ commenced 5 and $21 \mathrm{~min}$ into 28-min sessions and terminated with shock onset. During FOC Days 4-7, $\mathrm{CS}_{1}$ commenced 9 and $25 \mathrm{~min}$ into $30-\mathrm{min}$ sessions. On FOC 
Day $8, \mathrm{CS}_{1}$ commenced 7 and $25 \mathrm{~min}$ into the $30-\mathrm{min}$ session.

$\mathrm{CS}_{2}$ preexposure occurred during FOC Days 7 and 8. The flashing light was presented once each day, after 29 min into Day 7's session (following FOC trials) and $15 \mathrm{~min}$ into Day 8's session (between FOC trials, $7 \mathrm{~min}$ after the first US offset and $10 \mathrm{~min}$ before the second $\mathrm{CS}_{1}$ onset).

Bar us no-bar SOC. SOC began the day following FOC Day 8. After the Ss were assigned randomly to two equal groups balanced for "box" and "time of day" effects, they were given two light $\left(\mathrm{CS}_{2}\right)$-noise $\left(\mathrm{CS}_{1}\right)$ presentations daily for 3 days under differing conditions of response (barpressing) and reinforcement (water) availability. One group, Group B, had the bar present and water available on the original response-contingent basis. The other, Group NB, had the bar removed and water unavailable. On these trials, $\mathrm{CS}_{2}$ commenced 8 and $24 \mathrm{~min}$ into 30-min sessions, terminating each time with $\mathrm{CS}_{1}$ onset.

SOC test phase. Beginning with SOC Day 4, both groups were given 7 additional SOC days with the bar present and water response-contingent. The temporal relationships between $\mathrm{CS}_{2}, \mathrm{CS}_{1}$, and session length remained identical to those established for SOC Days 1-3.

\section{Suppression Index}

Suppression was indexed by the commonly used ratio $B /(A+B)$, where $B$ represents the number of barpresses during the $C S$ in question and $A$, the number during the 1-min pretrial interval. Daily FOC and $S O C$ suppression ratios were calculated for each $\mathbf{S}$ (where the bar was present) by averaging the two ratios obtained during each session.

Results and Discussion

Figure 1 displays the acquisition and extinction curves for $\mathrm{CS}_{1}$ and $\mathrm{CS}_{2}$. As may be seen, second-order conditioned suppression was readily obtained for both groups. A 2 (groups) by 2 (trials) analysis of variance of CS, suppression during preexposure (FOC Days 7 and 8 ) revealed only a significant effect of trials $[F(1,16)=4.79, p<.05]$, indicating similar reductions in suppression across trials for both groups. The combined mean $\mathrm{CS}_{2}$ suppression ratio on Day 8 was .36. During SOC Days 1-3, when the bar was present only for Group B, mean suppression ratios decreased progressively from .46 on the first trial of Day 1 to .34 on Day 3. A 2 (groups) by 7 (days) analysis of variance of $\mathrm{CS}_{2}$ suppression on SOC Days 4-10 also revealed only a significant days effect $[F(6,96)=5.98$, $\mathrm{p}<.001]$, representing similar SOC curves for the groups. Combined mean suppression ratios increased from .31 on SOC Day 4 to .39 on Day 10 , representing extinction to $\mathrm{CS}_{2}$.

Analyses of operant response rates during the last 3 days of preliminary training and pretrial intervals on SOC Day 4 further indicated no differences between groups. A 2 (groups) by 3 (days) analysis of variance of barpressing rates on Preliminary Operant Training Days 4-6 yielded no significant effects, indicating similar and stable operant responding for the groups prior to FOC. The overall mean a cross Days 4.6 was 9.0 barpresses/min. A 2 (groups) by 2 (trials) analysis of variance of response rates during the pretrial intervals on SOC Day 4 also revealed no significant effects. The overall mean for these two trials was 10.1 barpresses $/ \mathrm{min}$.

Finally, analyses of $\mathrm{CS}_{1}$ suppression again revealed no differences between groups, either during FOC or SOC. A 2 (groups) by 9 (days) analysis of variance of the FOC acquisition data yielded only a significant days effect $[F(8,128)=93.29, \quad p<.001]$, reflecting similar acquisition of first-order conditioned suppression for both groups. Without preexposure, $\mathrm{CS}_{1}$ produced a combined mean suppression ratio of .50 on FOC Trial 1, after which it quickly diminished and stabilized near .00

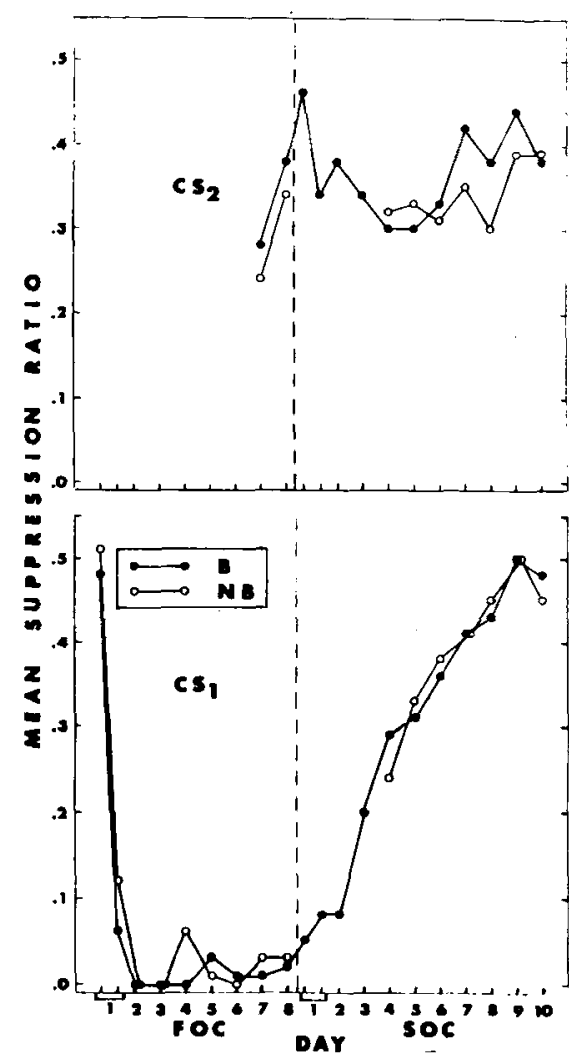

Fig. 1. Mean $\mathrm{CS}_{1}$ (noise) and $\mathrm{CS}_{2}$ (light) suppression ratios during $\mathrm{FOC}$ and SOC.
(.00-.03 on FOC Days 2-8). A 2 (groups) by 7 (days) analysis of variance of $\mathrm{CS}_{1}$ suppression during SOC similarly yielded only a significant days effect $[F(6,96)=21.37, p<.001]$, reflecting similar first-order extinction curves for both groups. The combined mean suppression ratios increased progressively from .26 on SOC Day 4 to .50 on Day 9.

$\mathrm{CS}_{1}$ and $\mathrm{CS}_{2}$ suppression performances were clearly well correlated during SOC, indicating that response availability did not differentiate Groups B and NB with respect to either extinction to $\mathrm{CS}_{1}$ or acquisition and extinction to $\mathrm{CS}_{2}$. Kamil's response-contingent counterconditioning hypothesis (1969) is therefore not supported by these results. Similarly, these results are contrary to those obtained by Jackson (1970) and Byrum \& Jackson (1970), since there was no treatment effect during first-order extinction to $\mathrm{CS}_{1}$. It appears, then, that response availability has no effect on acquisition of SOC.

\section{EXPERIMENT 2}

There were, however, several potentially significant methodological differences between the present study and those reported by Kamil (1969), Jackson (1970), and Byrum \& Jackson (1970). Kamil maintained his Ss at a relatively high deprivation level throughout experimentation ( $80 \%$ of their preexperimental weight), while the others allowed their Ss to surpass preexperimental weights prior to initial CER extinction (treatment). Experiment 1 involved high deprivation similar to that employed by Kamil: Ss returned to only $85 \%$ of preexperimental weights prior to SOC Day 1 (the first bar vs no-bar day). It may be that relatively high deprivation will overcome the effects of response availability during SOC.

Davenport (1966) noted that SOC was stronger under conditions of (1) high US intensity $(3.0 \mathrm{~mA}$ vs $0.5 \mathrm{~mA}$ ), (2) auditory vs visual $\mathrm{CS}_{1}$, and (3) distributed conditioning trials (one per day). Kamil (1969) used a 2.5-mA shock, an auditory $\mathrm{CS}_{1}$, and one trial per day. In doing so, he may have created a situation where his independent variables became relatively ineffectual as differential determinants of SOC. While Experiment 1 employed high deprivation and an auditory $\mathrm{CS}_{1}$, shock intensity was relatively low $(1.0 \mathrm{~mA})$, and trials were massed (two per day). Since no response availability effect was found under these conditions, Experiment 2 was designed to further weaken $S O C$ and increase the possibility of demonstrating a response availability effect. 
Method

Experiment 2 utilized 16 experimentally naive male Sprague-Dawley rats weighing an average of $417 \mathrm{~g} \quad(\mathrm{~s}=32.4)$ at the beginning of experimentation. The procedure was the same as in Experiment 1 except for the following alterations in the controlling variables: (1) water was available for $15 \mathrm{~min}$, rather than $5 \mathrm{~min}$, beginning $10 \mathrm{~min}$ after the conclusion of each daily 28-/30-min session; (2) $\mathrm{CS}_{1}$ became the 1-min $25 \cdot \mathrm{W}$ light flashing at $1.5 \mathrm{fps}, \mathrm{CS}_{2}$ the 1-min clicker; and (3) the bar vs no-bar initial SOC phase was lengthened from 3 to 5 days.

Results

Figure 2 displays the acquisition and extinction curves for $\mathrm{CS}_{1}$ and $\mathrm{CS}_{2}$. Similar second-order conditioned suppression curves were again obtained for both groups. A 2 (groups) by 2 (trials) analysis of variance of $\mathrm{CS}_{2}$ suppression during preexposure revealed no significant effects. The combined mean $\mathrm{CS}_{2}$ suppression ratio on FOC Day 8 was .53 , indicating complete habituation by both groups. During the bar vs no-bar SOC phase, mean suppression ratios for Group B decreased progressively from .51 on Day 1 to .40 on Day 5. A 2 (groups) by 5 (days) analysis of variance of $\mathrm{CS}_{2}$ suppression on SOC Days 6-10 revealed only a significant days effect $[F(4,56)=2.73, \quad p<.05]$, representing similar SOC curves for both groups. Combined mean suppression ratios increased from .46 on Day 6 to .51 on Day 10 , indicating extinction of SOC.

Analyses of CS, suppression ratios again revealed no differences between groups. A 2 (groups) by 9 (days) analysis of variance of FOC ratios yielded only a significant days effect $[F(8,112)=29.90, p<.001]$. Without preexposure, $\mathrm{CS}_{1}$ produced a combined mean suppression ratio of .34 on FOC Trial 1, after which it quickly diminished to and stabilized near .00. A 2 (groups) by 5 (days) analysis of variance of $\mathrm{CS}_{1}$ suppression during SOC Days $6 \cdot 10$ yielded no significant effects, indicating that first-order extinction was slow. Prior to SOC Day 6, the mean suppression ratio for Group $B$ had reached only .24. The combined mean suppression ratios across SOC Days 6-10 were .35, $.43, .44, .42$, and .43 , respectively, reflecting incomplete first-order extinction after 10 days.

Analyses of operant response rates indicated no differences between treatments. Responding was both similar and stable for the groups during the last 3 operant stabilization days and the pre-CS periods on SOC Day 6. The overall mean across stabilization Days 4-6 was 12.2

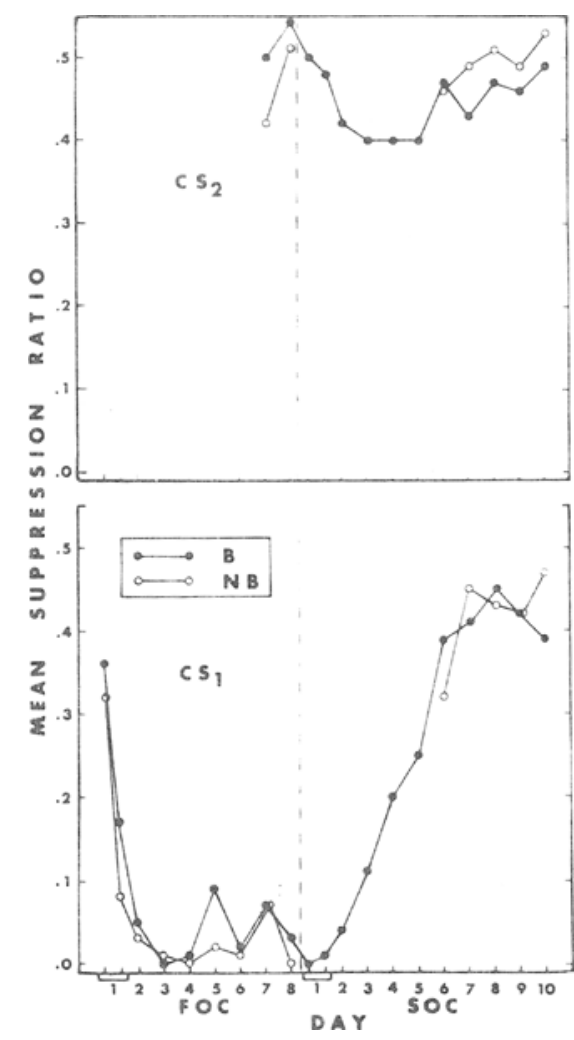

Fig. 2. Mean $\mathrm{CS}_{1}$ (light) and $\mathrm{CS}_{2}$ (noise) suppression ratios during $\mathrm{FOC}$ and SOC.

barpresses $/ \mathrm{min}$. The overall mean for the pre-CS periods on SOC Day 6 was 12.5 barpresses $/ \mathrm{min}$. Discussion

The results of Experiment 2 also provide evidence which clearly contradicts the response availability hypothesis. Despite decreased motivation (Ss surpassed preexperimental weights prior to $\mathrm{SOC})$, the use of a visual $\mathrm{CS}_{1}$, and a lengthened treatment period, neither SOC nor first-order extinction was differentially affected by the response-availability factor. With the exception of one major difference, Experiment 2 produced results almost identical to those of Experiment 1 .

This major difference is related to Davenport's (1966) original finding comparing visual and auditory stimuli. He emphasized "... (c) the rapidity with which second-order conditioning may be obtained with an auditory $\mathrm{CS}_{1}$, and (d) the relative difficulty of obtaining higher-order conditioning when the second-occurring $\mathrm{CS}$ is a visual stimulus...." The present studies readily obtained second-order conditioning with both auditory and visual $\mathrm{CS}_{1} \mathrm{~s}$.' It should be noted, however, that there was a distinct difference between the two; the visual stimulus was more aversive than the auditory stimulus. Upon tirst presentation, either during preexposure (as $\mathrm{CS}_{2}$ ) or the first trial of FOC (as CS, ), the visual stimulus produced greater suppression than the auditory stimulus. Furthermore, habituation and extinction (either first- or second-order) were slower for the visual CS than for the auditory CS.

A final problem relates to Jackson's (1970) finding of more rapid extinction for $S$ s under the response-availability conditioning during early CER extinction. Based on Jackson's results, $S s$ in the present studies who had the bar present during early SOC should have acquired SOC less rapidly or less completely because of more rapid extinction to the first-order stimulus. There was, however, neither more rapid extinction for Ss under the response-availability condition during early CER extinction. Based on while Jackson (1970) gave CER training with the bar removed, the present studies employed the bar throughout FOC. Perhaps bar absence during CER acquisition may be necessary before the response availability effect during early extinction can occur. A 2 (bar vs no bar during acquisition) by 2 (bar vs no bar during early extinction) design should provide the answer to this question. Alternatively, if the response availability effect is reliable, regardless of first-order acquisition procedures, there may be something about SOC that interacts with this effect to cancel its expression. This possibility can be evaluated by extending the present studies to include both bar and no-bar conditions during FOC.

\section{REFERENCES}

BYRUM, R. P., \& JACKSON, D. E. Reinforcement and response factors in the extinction of conditioned suppression. Psychonomic Science, 1970, 20, 143-144.

DAVENPORT, J. W. Higher-order conditioning of fear (CER). Psychonomic Science, 1966, 4, 27-28.

JACKSON, D. E. Response availability and extinction of conditioned suppression. Psychonomic Science, 1970, 19, 160.

KAMIL. A. C. The second-order conditioning of fear in rats. Psychonomic Science, 1968, 10, 99-100.

KAMIL, A. C. Some parameters of the second-order conditioning of fear in rats. Journal of Comparative \& Physiological Psychology, 1969, 67, 364-369.

WAGNER, A. R., SIEGEL, L. S., \& FEIN, G. G. Extinction of conditioned fear as a function of percentage of reinforcement. Journal of Comparative \& Physiological Psychology, 1967, 63, 160-164. 